\title{
Ophthalmic complications of HIV/AIDS
}

\author{
Frank G Ah-Fat, Mark Batterbury
}

\begin{abstract}
Summary
As a result of improved treatment and patient survival, ophthalmic complications are now being seen with increasing frequency in AIDS, occurring in up to $75 \%$ of patients during the course of the disease. The eye may be involved by an AIDS-related microvasculopathy, which gives rise to cotton wool spots, and by opportunistic infections caused by a wide range of organisms, including cytomegalovirus, herpes simplex virus, varicella zoster, Toxoplasma gondii, Mycobacterium avium-intracellulare, Treponema pallidum, Pneumocystis carinii and various fungal agents. Opportunistic infections may be the presenting sign of disseminated infection. The eye may also be involved by neoplasms such as Kaposi's sarcoma and lymphoma, and by intracranial disease. Ocular involvement may lead to blindness if untreated and prompt ophthalmological referral is essential. This article reviews the range of ocular diseases seen in HIV and AIDS, current therapeutic options and outcome.
\end{abstract}

Keywords: HIV, AIDS, ophthalmic complications

St Paul's Eye Unit, Royal Liverpool University Hospital, Prescot Street, Liverpool L7 8XP, UK

FG Ah-Fat

M Batterbury

Accepted 24 January 1996
Since it was first described in 1981, acquired immune deficiency syndrome (AIDS) has become a cause of major public health concern worldwide. With the increasing use of antiretroviral drugs, as well as improved prophylaxis and treatment of opportunistic infections (in particular, Pneumocystis carinii pneumonia), the survival of patients with AIDS has increased. As a result, other forms of opportunistic infection, including those involving the eye, are now being seen with increasing frequency. ${ }^{1,2}$ Ocular complications can be seen in up to $75 \%$ of patients in the course of the disease and are potentially sightthreatening. Many of these complications were previously seen only rarely in general ophthalmic practice, but they now present a formidable challenge to the ophthalmologist. They may show an atypical clinical appearance, follow an unusual course, and not infrequently prove refractory to conventional treatment.

The ophthalmic manifestations of AIDS can be grouped into four main categories: microvascular disease, opportunistic infections, neoplasms, and the neuro-ophthalmic manifestations of intracranial disease (box 1). Ophthalmic disease may affect any part of the visual system, namely the ocular adnexae (eyelids and orbit), the anterior segment (conjunctiva, cornea and iris), the posterior segment (retina, choroid and vitreous) and the optic nerve and afferent pathways. An anatomical approach is useful in the understanding and assessment of visual loss (box 2).

\section{Ocular adnexal disease}

\section{KAPOSI'S SARCOMA}

The ocular adnexae may be involved by neoplasms such as Kaposi's sarcoma or lymphoma. Ocular Kaposi's sarcoma usually presents as a red mass on the eyelid or in the conjunctiva of the lower fornix, where it is easily mistaken for a subconjunctival haemorrhage. ${ }^{3,4}$ Involvement of the orbit may give rise to proptosis and diplopia. Kaposi's sarcoma in AIDS tends to be more resistant to treatment than endemic Kaposi's sarcoma. Vinca alkaloids (principally vinblastine), daunorubicin and bleomycin are the most commonly used chemotherapeutic agents. The use of alpha- and beta-interferon has met with limited success. Radiotherapy on the other hand has been found to be both safe and effective in local palliation. ${ }^{3}$

\section{OCULAR LYMPHOMA}

Lymphoma of the eyelids and of the orbit is generally of the high grade B-cell type or varieties similar to Burkitt's lymphoma. They present as lumps affecting the eyelids and the conjunctiva or arise deeper in the orbit, causing proptosis and diplopia. ${ }^{5}$ The vitreous and retina may also be involved by primary ocular lymphoma. Patients need to be investigated for systemic lymphoma with imaging and biopsy. Treatment is mainly in the form of chemotherapy and radiotherapy.

\section{MOLLUSCUM CONTAGIOSUM}

Molluscum contagiosum, caused by a pox virus, may appear during the course of HIV infection. It is characterised by multiple small elevated eyelid nodules which have an umbilicated central core (figure 1). Ocular irriation may result from an associated follicular keratoconjunctivitis. Molluscum has a tendency to recur after surgical removal or cryotherapy. ${ }^{6}$

\section{Conjunctival and corneal disease}

\section{CONJUNCTIVITIS AND KERATITIS}

A mild and transient conjunctivitis is common amongst AIDS patients but it does not usually lead to serious ocular sequelae. ${ }^{4}$ Conjunctival vascular sludging may be seen on slit-lamp examination as a result of a microvasculopathy associated with AIDS or human immunodeficiency virus (HIV) infection.? 


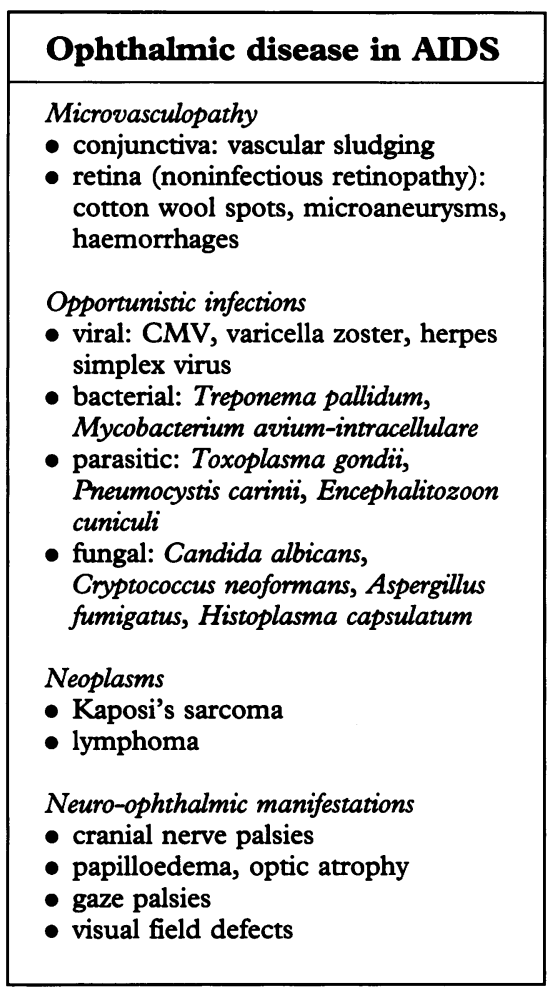

Box 1

\begin{tabular}{|l|}
\hline Causes of visual loss in AIDS \\
\hline - corneal disease (eg, herpes simplex, \\
varicella zoster \\
- uveitis (eg, toxoplasmosis, syphilis) \\
- retinal disease (eg, CMV, \\
toxoplasmosis) \\
- optic neuropathy (eg, syphilis, \\
lymphoma) \\
\hline
\end{tabular}

Box 2
Keratoconjunctivitis sicca is a further recognised feature of AIDS. ${ }^{8}$ The lack of an adequate tear film may predispose such patients to sight-threatening bacterial corneal ulcers. Dendritic ulcers caused by herpes simplex virus are no more common amongst AIDS sufferers, but are unusual in that the peripheral rather than the central cornea is affected. These ulcers may follow a protracted course and recur more frequently despite antiviral treatment. ${ }^{9}$

\section{OPHTHALMIC HERPES ZOSTER}

Ophthalmic herpes zoster is a recognised complication in both the early and the late stages of HIV infection. ${ }^{10}$ It is caused by reactivation of varicella zoster infection and is commonly associated with a keratitis or an anterior uveitis. Ocular inflammation can be severe and prolonged, leading to cataract and glaucoma. In the presence of immunosuppression, disseminated zoster may develop. It is not unusual for the disease to follow a protracted course and early diagnosis and treatment with systemic acyclovir is important to prevent visual loss.

\section{CORNEAL MICROSPORIDIOSIS}

Corneal microsporidiosis is a recently described condition amongst AIDS patients. ${ }^{11}$ It is caused by the protozoal parasite Encephalitozoon cuniculi, and its features comprise a conjunctivitis as well as a painful chronic epithelial keratitis. Brolene (dibromopropamidine), itraconazole and fumagillin have been found to be effective in providing symptomatic relief.

\section{Non-infectious retinopathy}

The most common ocular manifestation of HIV disease is a non-infectious retinopathy that occurs secondary to microvascular disease (box 3). It is usually bilateral and consists of cotton wool spots, intraretinal haemorrhages, microaneurysms and Roth spots (retinal haemorrhages with a white centre). ${ }^{4,7,8,12}$ Clinical and autopsy examinations have revealed the presence of cotton wool spots in nearly $75 \%$ of patients. ${ }^{4,13}$ They appear as fluffy white lesions identical to those seen in diabetes and hypertension (figure 2). Cotton wool spots represent small nerve fibre layer infarcts secondary to a microvasculopathy. They are not caused by direct infection of the retina and should therefore not be mistaken for cytomegalovirus retinitis. ${ }^{12}$ Cotton wool spots appear intermittently during the course of HIV infection, resolving spontaneously after a period of six to eight weeks without serious visual sequelae. This contrasts with cytomegalovirus retinitis which, if untreated, invariably progresses to blindness.

\section{Infectious retinopathy}

Viral, fungal, mycobacterial and protozoal infections of the retina, choroid or vitreous pose the greatest challenge to the clinician. Many of these infections were previously only rarely seen in general ophthalmic practice. Diagnoses are often made purely on the clinical appearance or at autopsy. Serological tests are not reliable in immunocompromised individuals. A positive titre may simply denote previous infection, whilst a negative titre does not rule out active infection. Ocular involvement needs to be regarded as part of systemic disease. In some cases, ocular infections may be the presenting sign of disseminated disease.

\section{CYTOMEGALOVIRUS RETINITIS}

Prior to the AIDS epidemic, cytomegalovirus (CMV) retinitis was seen only in renal transplant recipients, in patients receiving immunosuppressive chemotherapy for lymphoma and leukaemia and in the newborn with cytomegalic inclusion disease. It is now the most common opportunistic infection in AIDS, with autopsy studies showing that over a third of patients are affected. ${ }^{13}$ While CMV retinitis is an AIDS-defining diagnosis, it is normally seen only in the later stages of AIDS, when the CD4 count falls below 50 cells $/ \mathrm{mm}^{3} \cdot{ }^{14} \mathrm{CMV}$ retinitis follows a relentless course and invariably progresses to blindness if untreated. Its onset is associated with few visual symptoms initially. As unilateral disease may go unnoticed until the patient occludes the unaffected eye, or develops bilateral involvement, the disease may already be advanced at the time of diagnosis. ${ }^{15}$ Symptoms include floaters (caused by vitreal inflammation), a shimmering effect, shadows and blurring. The fundal appearance is characteristic, consisting of white granular lesions similar to cotton wool spots, but with better defined borders. Lesions start adjacent to major blood vessels in the peripheral retina

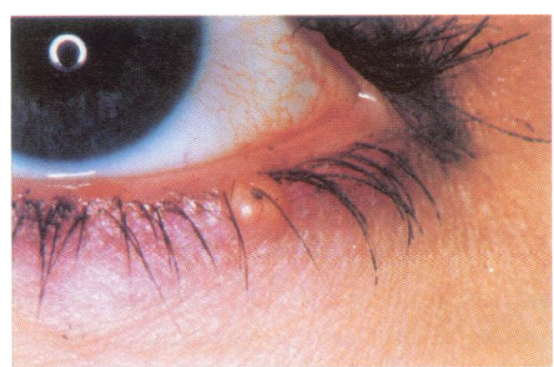

Figure 1 Molluscum contagiosum of the lower lid 


\begin{tabular}{|l|}
\hline Retinal disease in AIDS \\
\hline HIV retinopathy (noninfectious \\
retinopathy) \\
- cotton wool spots \\
- haemorrhages \\
- microaneurysms \\
- Roth spots \\
CMV retinitis \\
- retinal whitening and opacification \\
with haemorrhages \\
- lesions adjacent to major blood \\
vessels \\
- lesions spread from initial focus and \\
become confluent \\
- retinal necrosis, atrophy and \\
detachment \\
- lack of marked inflammation or \\
vitritis \\
Toxoplasmosis \\
- dense white retinochoroiditis, not \\
centred along blood vessels \\
- multifocal and not adjacent to old \\
lesions \\
- healed areas become densely \\
pigmented \\
- marked inflammation (iridocyclitis \\
or vitritis) \\
Acute retinal necrosis \\
- inflammatory reaction with vitritis \\
- yellow-white confluent retinal \\
necrosis, scanty haemorrhages \\
- sharp demarcation between normal \\
and abnormal retina \\
- vascular occlusion \\
Syphilitic retinitis \\
- marked inflammation (iridocyclitis \\
or vitritis) \\
- retinitis, choroiditis, papillitis \\
\hline
\end{tabular}

Box 3

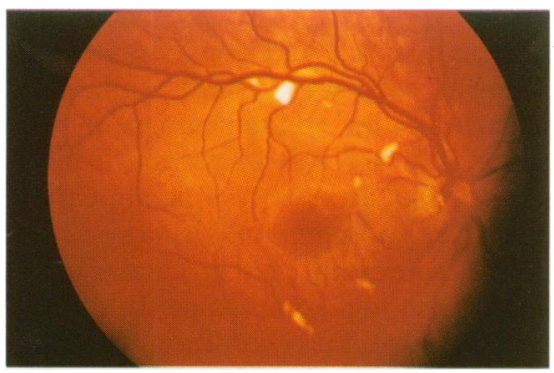

Figure 2 Cotton wool spots (noninfectious retinopathy)

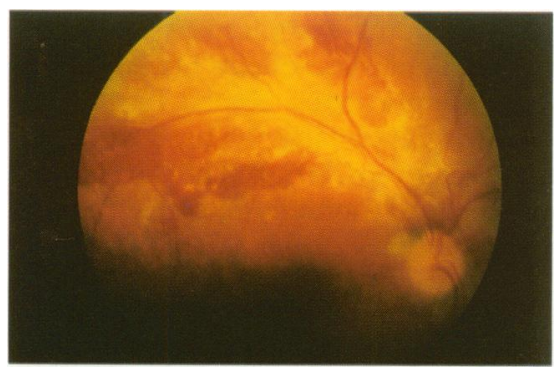

Figure 3 Cytomegalovirus retinitis or in the macular area and are associated with perivascular retinal haemorrhages and vessel sheathing (figure 3). ${ }^{8}$ The lesions enlarge centrifugally, and clinical appearances in florid cases have been likened to a 'tomato sauce and salad dressing' fundus. Vision is lost because of macular involvement or retinal detachment secondary to full thickness retinal necrosis. Optic atrophy may occur as a result of retinal disease and optic neuritis. Unlike other forms of necrotising retinitis, marked ocular inflammation is not seen and the eye usually appears white externally. Serology is not helpful in the diagnosis in the immunocompromised host. ${ }^{12}$ A positive titre may simply indicate past infection whilst a negative titre does not rule out infection. CMV retinitis is diagnosed by its typical fundal appearance. It needs to be distinguished, however, from toxicoplasmosis and syphilitic retinitis, both of which give rise to marked inflammatory reactions, and from acute retinal necrosis, which shows a more rapid progression (box 3). Buffy coat culture of blood and urine are needed as CMV retinitis may be the presenting sign of tissue-invasive infection affecting the central nervous system, the gastrointestinal tract, the respiratory tract and the adrenals.

\section{Treatment of cytomegalovirus retinitis}

Ganciclovir and foscarnet are the two main agents currently available for the treatment of CMV retinitis. ${ }^{16,17}$ As neither agent eliminates the virus from the eye, treatment needs to be life-long. Insertion of a long-term indwelling central venous catheter is required, since both agents are administered intravenously. Treatment consists of an induction course of two or three weeks, followed by a lower maintenance dose (box 4). Regular follow-up is essential. Relapses are common and necessitate treatment with a further induction dose.

Ganciclovir Ganciclovir is a guanine analogue which inhibits viral DNA replication. An induction course of $5 \mathrm{mg} / \mathrm{kg}$ twice daily for $14-21$ days is followed by a maintenance dose of $5 \mathrm{mg} / \mathrm{kg}$ daily. The main side-effect of ganciclovir is bone marrow suppression, which occurs in up to $38 \%$ of patients. ${ }^{16}$ Concurrent zidovudine therapy may therefore be difficult. Treatment often has to be suspended if the neutrophil count falls below $500 / \mathrm{mm}^{3}$. However, the introduction of granulocyte growth factors such as Sargramostim (granulocyte-monocyte colony-stimulating factor) and Filgrastim (granulocyte colony-stimulating factor) and the use of alternative antiretroviral agents (eg, dideoxyinosine) has improved the outlook for many patients. ${ }^{18,19}$

Foscarnet Foscarnet is a pyrophosphate analogue that inhibits DNA polymerase of cytomegalovirus. It also has some antiretroviral activity. As bone marrow suppression is much less marked, concurrent use of zidovudine is possible. It is given as an induction dose of $90 \mathrm{mg} / \mathrm{kg}$ bid for two to three weeks followed by a maintenance dose of $120 \mathrm{mg} / \mathrm{kg}$ once daily. The main side-effect of foscarnet is nephrotoxicity, and although this can be minimised by pretreatment with saline loading, it often requires the patient to switch to ganciclovir. Patients may develop further intolerance to foscarnet in the form of seizures and electrolyte abnormalities (hypocalcaemia, hypokalaemia, hypomagnesaemia and hypophosphataemia). Compared with ganciclovir, patients on foscarnet are also more likely to develop infusion-related symptoms (such as nausea and parasthesia) and genito-urinary ulcers. ${ }^{20}$

Ganciclovir vs foscarnet A multicentre controlled trial conducted by the Studies of the Ocular Complications of AIDS Research Group in collaboration with the AIDS Clinical Trials Group to compare ganciclovir with foscarnet has shown similar efficacies for the two drugs in terms of end-points such as interval to first reactivation and progression (56 days in the ganciclovir group and 59 days in the foscarnet group) as well as visual acuity. However, patients on foscarnet were found to have a median survival of 12.6 months, compared to 8.5 months for those on ganciclovir. ${ }^{21}$ While it is possible that the differences in survival were related to the intrinsic antiretroviral activity of foscarnet or to the fact that patients on ganciclovir were less able to tolerate zidovudine, the Policy and Data Monitoring Board recommended suspension of the treatment protocol after 19 months. Patients on foscarnet, however, were more likely to switch treatment as a result of toxic side-effects. The choice of treatment needs to be made ultimately on an individual basis, taking into account the patient's tolerance and motivation.

Intravitreal ganciclovir Intravitreal ganciclovir has been advocated for patients unable to tolerate systemic treatment, either because of toxicity or infusion- 


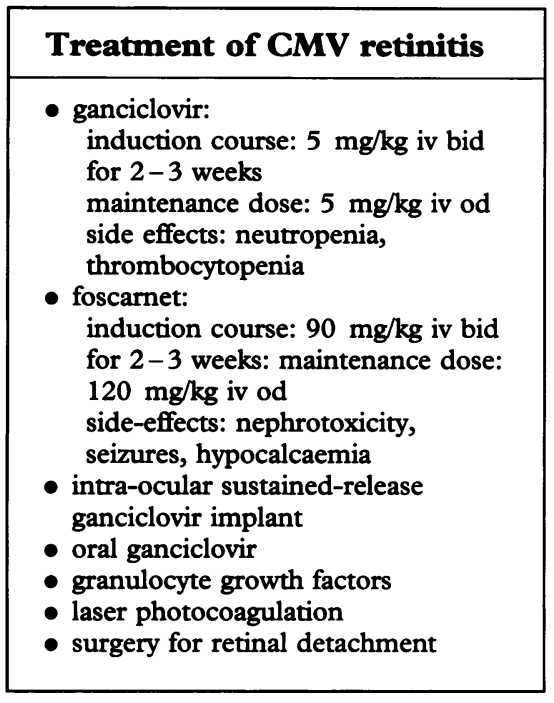

Box 4
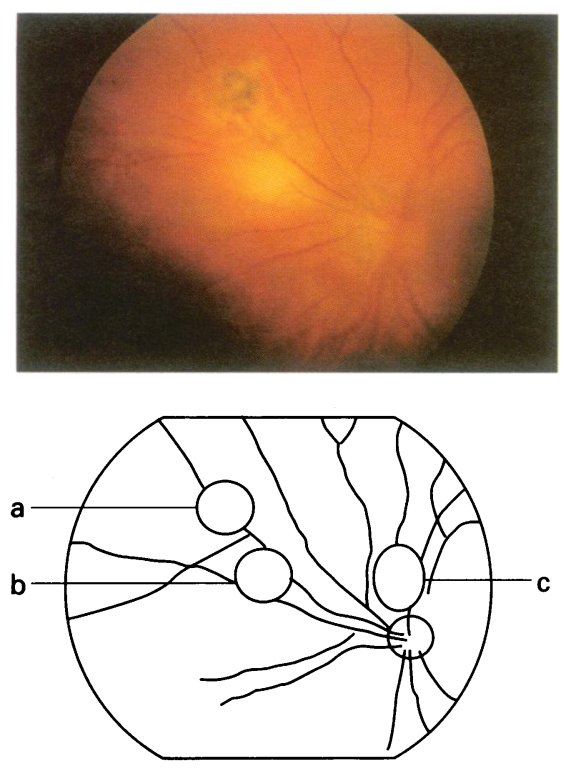

Figure 4 Ocular toxoplasmosis. The line diagram identifies (a) an old pigmented scar, (b) a fresh focus of infection, and (c) a vitreous haze related problems. Recent interest has centred on a sustained-release device that maintains high levels of the drug in the eye. ${ }^{22}$ However, this option must be weighed against its potential disadvantages, which include the risk of endophthalmitis and retinal detachment as well as the loss of prophylaxis against disease elsewhere in the gastrointestinal tract, central nervous system and in the other eye.

Oral ganciclovir An oral preparation of ganciclovir has recently become available and has the advantage of avoiding the problems associated with the use of an intravenous catheter. Oral ganciclovir is, however, limited by poorer bioavailability. The lower serum levels achieved may make viral resistance more likely and lead to earlier relapse. It may find a future role as a prophylactic agent. $^{2}$

Other treatment options Retinal detachment may complicate the later stages of CMV retinopathy. Whilst surgery may be successful in achieving retinal reattachment, visual outcome has been disappointing owing to optic nerve disease and retinal damage. Laser photocoagulation to create a barrier chorioretinal scar has not been found to be effective in preventing progression of CMV retinitis. It may, however, find a role as treatment or prophylaxis for retinal detachment. Patients who develop a relapse of CMV retinitis have traditionally been treated with a further induction course of either ganciclovir or foscarnet. The Cytomegalovirus Retreatment Trial has shown combination therapy to be more effective than monotherapy in controlling recurrent disease. ${ }^{23}$ Several drugs are also presently under investigation. Cidofovir has a broad spectrum activity against herpes viruses but is limited by renal toxicity. ISIS 2922 , given intravitreally, inhibits the messenger RNA of CMV. A monoclonal CMV antibody is also currently undergoing trials as adjunctive therapy. ${ }^{2}$

\section{ACUTE RETINAL NECROSIS}

Acute retinal necrosis is a rare necrotising retinitis typically affecting healthy immunocompetent individuals of any age, but is now also seen in patients with AIDS. It is caused by viruses of the herpes group, most commonly the varicella zoster virus but also the herpes simplex virus and CMV. ${ }^{24}$ Patients may give a history of recent cutaneous zoster and complain of peri-orbital pain and blurred vision. Unlike CMV retinitis, intense inflammation is a common feature. Fundal changes include sheathing of arterioles with minimal haemorrhages and multiple yellow - white retinal lesions which become confluent and progress to full thickness necrotising retinitis. The disease becomes bilateral in $40 \%$ of cases. Systemic acyclovir or ganciclovir have been used to arrest the disease process, but the overall prognosis is poor, especially if there is an associated retinal detachment. ${ }^{12}$

\section{OCULAR TOXOPLASMOSIS}

Toxoplasma gondii is a common opportunistic pathogen in AIDS patients and it affects many organs, particularly the central nervous system and, less commonly, the eye. ${ }^{25}$ In healthy individuals, ocular toxoplasmosis is the commonest cause of posterior uveitis, occurring as a result of reactivation of encysted organisms following intra-uterine infection. Retinal lesions appear as white infiltrates which later take the appearance of pigmented scars as the inflammation subsides (figure 4). A vitritis is common, and gives rise to a vitreous haze or in severe cases to a 'headlight in the fog' appearance. In AIDS, the disease is unusual in many ways. Bilateral involvement is common, with the appearance of multifocal lesions that are not adjacent to old scars (box 3). These may therefore represent cases of newly acquired toxoplasmosis rather than reactivation. The organism also appears to affect both the retina and the choroid. ${ }^{25}$ Antibody titres are not helpful in the diagnosis. A rising IgG antibody titre may not be detected and IgM antibody may be absent in AIDS. Potentially life-threatening cerebral involvement is frequent in the presence of ocular disease. Agents available for the treatment of toxoplasmosis include pyrimethamine, sulphadiazine and clindamycin. Toxic side-effects are common and include marrow suppression and pseudomembranous colitis. Prolonged treatment may be required to prevent new lesions developing. ${ }^{25}$ There has been much interest recently in the drug atovaquone $(566 \mathrm{C} 80){ }^{26}$ This drug was originally synthesised as an antimalarial, but has been found to be active against Toxoplasma gondii cysts, as well as against Pneumocystis carinii.

\section{Pneumocystis carinii CHOROIDITIS}

$P$ carinii choroiditis is a new ophthalmic disorder which had not been described prior to the emergence of AIDS. Affected patients often have a history of 


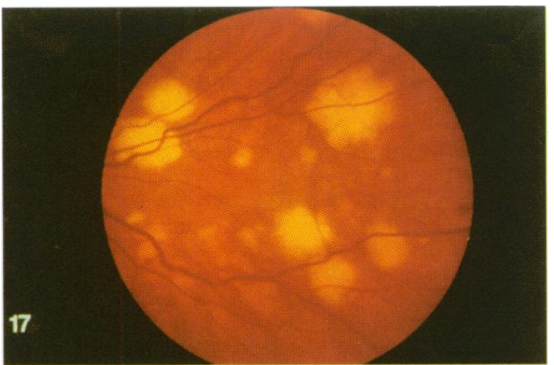

Figure 5 Pneumocystis carinii choroiditis

\begin{tabular}{|l|}
\hline Ocular syphilis \\
\hline Primary syphilis \\
- chancre of lids/conjunctiva \\
Secondary syphilis \\
- conjunctivitis \\
- episcleritis/scleritis \\
- interstitial keratitis \\
- dacryoadenitis/dacryocystitis \\
- anterior uveitis (iritis) \\
chosterior uveitis incl vitritis and \\
- neuroiditis \\
- papillitis \\
- retrobulbar neuritis \\
Tertiary syphilis \\
- cranial nerve palsies \\
- ptosis \\
- optic atrophy \\
- Argyll Robertson pupil \\
\hline
\end{tabular}

Box 5 aerosolised pentamidine treatment or prophylaxis. This route of drug delivery does not provide protection against extrapulmonary $P$ carinii infection and it may even encourage disseminated infection. ${ }^{27}$ The use of oral septrin instead of aerosol prophylaxis may be preventative. $P$ carinii choroiditis appears as multiple, pale yellow plaque-like lesions in the fundus with no associated intraocular inflammation (figure 5). The condition may respond to systemic treatment with intravenous trimethoprim-sulphamethoxazole or pentamidine. Though the patient may be visually asymptomatic, $P$ carinii choroiditis underscores the importance of regular ophthalmologic examination as it may be the first indication of disseminated infection in the presence of advanced HIV disease.

\section{BACTERIAL INFECTIONS}

The two most common bacterial organisms to affect the eye in AIDS are Treponema pallidum and Mycobacterium avium-intracellulare. Syphilis has seen a resurgence with AIDS. Ocular involvement is seen in both the early and the late stages of HIV infection..$^{28}$ Syphilis has been dubbed the 'great imitator' and its manifestations are protean (box 5). Unlike CMV retinitis, ocular syphilis causes more anterior segment inflammation in the form of an iridocyclitis, in addition to conjunctivitis and scleritis. Posterior segment disease causes a vitritis, retinitis, optic papillitis, retrobulbar neuritis and optic atrophy. Serology may not be helpful in the presence of immunosuppression. ${ }^{28}$ There is an associated high frequency of acute syphilitic meningitis or asymptomatic neurosyphilis as demonstrated on lumbar puncture. These may cause raised intracranial pressure with papilloedema and cranial nerve palsies. High dose intravenous penicillin $\mathrm{G}$ (12-24 MU daily for at least 10 days) has been found to be effective in the treatment of ocular syphilis and neurosyphilis. ${ }^{28}$ Mycobacterium avium-intracellulare infection of the eye is rarely seen in AIDS. It appears as a discrete choroidal granuloma from which the causative organism has been isolated at autopsy. ${ }^{13}$

\section{FUNGAL INFECTIONS}

While fungal infections are common in AIDS, ocular involvement is seen only occasionally. For example, Candida albicans infection commonly occurs in the gastrointestinal tract, but ocular candidiasis is rare. It occurs mainly in association with the use of indwelling catheters or with intravenous drug abuse. It causes a focus of chorioretinitis with an overlying vitreous haze. Cryptococcus neoformans is a well recognised cause of meningitis in ADIS. It may invade the eye and cause visual loss through choroiditis, retinitis and endophthalmitis. Cerebral involvement may also be associated with cranial nerve palsies and papilloedema ${ }^{29}$ Histoplasma capsulatum and Aspergillus fumigatus normally cause pulmonary infections, but isolated cases of ocular involvement have been reported in association with disseminated disease. ${ }^{30,31}$

\section{Neuro-ophthalmic manifestations}

Clinical evidence of intracranial disease is seen in up to $39 \%$ of AIDS sufferers. ${ }^{32}$ The central nervous system may be affected by neoplasms such as lymphoma and Kaposi's sarcoma or by opportunistic infections causing encephalitis, meningitis or cerebral abscess. Cranial nerve palsies occur as a result of raised intracranial pressure or secondary to an inflammatory or neoplastic neuropathy. They are most commonly associated with herpes zoster ophthalmicus, cryptococcal infection and lymphoma. Diplopia or pupillary abnormalities may be the first sign of serious intracranial disease. Gaze palsies and internuclear ophthalmoplegia have been seen in association with HIV encephalopathy, syphilis and toxoplasmosis. Papilloedema, optic neuritis, optic atrophy and visual field defects have also been described in relation to nervous system involvement by neoplasms or opportunistic infections.

\section{Conclusion}

The incidence of ocular disease in AIDS is likely to increase in the future as a result of improved patient survival. Ocular involvement may be the first indication of disseminated opportunistic infection. Moreover, the threat of sight loss is a major fear among AIDS sufferers. Early diagnosis and treatment of ocular complications are important to improve the quality of life. This requires a multidisciplinary approach to the management of AIDS and ophthalmological referral should be made at an early stage if ocular involvement is suspected. 
1 Hoover DR, Saah AJ, Bacellar H, et al. Clinical manifestations of AIDS in the era of Pneumocystis prophylaxis. N Engl $\mathcal{f}$ Med 1993; 329: $1922-6$.

2 Polis MA, Masur H. Promising new treatments for cytomegalovirus retinitis. $\mathfrak{f} A M A$ 1995; 273: 1457-9.

3 Shuler JD, Holland GN, Miles SA, Miller BJ, Grossman I. Kaposi sarcoma of the conjunctiva and eyelids associated with the acquired immunodeficiency syndrome. Arch Ophthalmol 1989; 107: 858-62.

4 Holland GN, Pepose JS, Pettit TH, Gottlieb MS, Yee RD, Foos RY. Acquired immune deficiency syndrome: ocular manifestations. Ophthalmology 1983; 90: 859-73.

5 Newsome DA. Noninfectious ocular complications of AIDS. Int Ophthalmol Clin 1989; 29: 95-7.

6 Robinson MR, Udell IJ, Garber PF, Perry HD, Streeten BW. Molluscum contagiosum of the eyelids in patients with acquired immune deficiency syndrome. Ophthalmology 1992; 99: 1745-7.

7 Engstrom RE, Holland GN, Hardy WD, Meiselman HJ. Hemorheologic abnormalities in paman infection and ophthalmic microvasculopathy. Am $\Im$ Ophthalmol 1990; 109: 153-61.

8 Khadem M, Kalish SB, Goldsmith J, et al. Khadem M, Kalish SB, Goldsmith J, et al. Ophthalmologic findings in acquired immunodeficiency syndrome (AIDS). Arch Ophthalmol 1984; 102: $201-6$.

9 Young TL, Robin JB, Holland GN, et al. Herpes simplex keratitis in patients with acquired immune deficiency syndrome. Ophthalmology 1989; 96: 1476-9.

10 Cole EL, Meisler DM, Calabrese LH, Holland GN, Mondino BJ, Conant MA. Herpes zoster ophthalmicus and acquired immune deficiency syndrome. Arch Ophthalmol 1984; 102: 1027 -9.

11 Friedberg DN, Stenson SM, Orenstein JM, Tierno PM, Charles NC. Microsporidial keratoconjunctivitis in acquired immunodeficiency toconjunctivitis in acquired immunodeficiency

12 Freeman WR, Lerner CW, Mines JA, et al. A Freeman WR, Lerner CW, Mines JA, et al. A
prospective study of the ophthalmologic findings in the acquired immune deficiency syndrome. Am $\mathcal{F}$ Ophthamol 1984; 97: 133-42.
13 Pepose JS, Holland GN, Nestor MS, Cochran AJ, Foos RY. Acquired immune deficiency syndrome: pathogenic mechanisms of ocular disease. Ophthalmology 1985; 92: 472-84

14 Pertel P, Hirschtick R, Phair J, Chmiel J, Poggensee L, Murphy R. Risk of developing cytomegalovirus retinitis in persons infected with the human immunodeficiency virus. $f$ AIDS 1992; 5: 1069-74.

15 Dhillon B. The management of cytomegalovirus retinitis in AIDS. Br fOphthalmol 1994; 78: 66-

16 Holland GN, Sidikaro Y, Kreiger AE, et al. Treatment of cytomegalovirus retinopathy with ganciclovir. Ophthalmology 1987; 94: 815-23.

17 Palestine AG, Polis MA, de Smet MD, et al. A randomised, controlled trial of foscarnet in the treatment of cytomegalovirus retinitis in patients with AIDS. Ann Intern Med 1991; 115: 665-73.

18 Hardy WD. Combined ganciclovir and recombinant human granulocyte-macrophage colonystimulating factor in the treatment of cytomega-
lovirus retinitis in AIDS patients. $\mathcal{f}$ AIDS 1991; 4 (suppl 1): s22-8.

19 Yarchoan R, Mitsuya H, Myers CE, Broder S Clinical pharmacology of zidovudine and related dideoxynucleosides. N Engl $f$ Med 1989; 321: $726-38$.

20 Studies of Ocular Complications of AIDS Research Group in collaboration with the AIDS Clinical Trials Group. Morbidity and toxic effects associated with ganciclovir or foscarnet therapy in a randomized cytomegalovirus retiniis trial. Arch Intern Med 1995; 155: 65-73.

21 Studies of Ocular Complications of AIDS Research Group in collaboration with the AIDS Clinical Trials Group. Mortality in patients with the acquired immunodeficiency syndrome treated with either foscarnet or ganciclovir for cytomegalovirus retinitis. $N$ Engl f Med 1992; 326: $213-20$.

22 Sanborn GE, Anand R, Torti RE, et al. Sustained-release ganciclovir therapy for treatment of cytomegalovirus retinitis. Use of an intravitreal device. Arch Ophthalmol 1992;110 $188-95$.
23 Studies of Ocular Complications of AIDS Research Group in collaboration with the AIDS Clinical Trials Group. Combination foscarnet and ganciclovir therapy vs monotherapy for the treatment of relapsed cytomegalovirus retinitis in patients with AIDS. Arch Ophthalmol 1996; 114: 23-33.

24 Freeman WR, Thomas EL, Rao NA, et al. Demonstration of herpes group virus in acute retinal necrosis syndrome. Am $\mathcal{f}$ Ophthalmol 1986; 102: 701-9.

25 Holland GN, Engstrom RE, Glasgow BJ, et al. Ocular toxoplasmosis in patients with the acquired immunodeficiency syndrome. $A m \mathcal{f}$ Ophthalmol 1988; 106: 653-67.

26 Lopez JS, de Smet MD, Masur H, Mueller BU, Pizzo PA, Nussenblatt RB. Orally administered $566 \mathrm{C} 80$ for treatment of ocular toxoplasmosis in a patient with the acquired immunodeficiency syndrome. Am f Ophthalmol 1992; 113: 331 - 3 .

27 Shami MJ, Freeman W, Friedberg D, Siderides E, Listhans A, Ai E. A multicentre study of Pneumocystitis choroidopathy. Am f Ophthalmol 1991; 112: 15-22.

28 McLeish WM, Pulido JS, Holland S, Culbertson WW, Winward $K$. The ocular manifestations of syphilis in the human immunodeficiency virus syphilis in the human immunodeficiency virus type-1 in $196-203$.

29 Crump JRC, Elner SG, Elner VM, Kauffman CA. Cryptococcal endophthalmitis: case report and review. Clin Infect Dis 1992; 14: 1069-73.

30 Macher A, Rodrigues MM, Kaplan W, et al. Disseminated bilateral chorioretinitus due to Histoplasma capsulatum in a patient with the acquired immunodeficiency syndrome. Ophthalmology 1985; 92: 1159-64.

31 Denning DW, Follansbee SE, Scolaro $M$, Edelstein H, Stevens DA. Pulmonary aspergillosis in the acquired immunodeficiency syndrome. $N$ Engl F Med 1991; 324: 654-62.

32 Levy RM, Bredesen DE, Rosenblum ML Neurological manifestations of the acquired immune deficiency syndrome (AIDS): experience at UCSF and review of the literature. $f$ Neurosurg 1985; 62: 475-95. 